\title{
An Inexpensive Digital Watermarking Scheme for Printed Document
}

\author{
Malay K. Kundu and Arpan K. Maiti \\ Machine Intelligence Unit, \\ Indian Statistical Institute, \\ Kolkata 700108 \\ Email: \{malay, arpan_t $\} @$ isical.ac.in
}

\begin{abstract}
This paper describes a method of hiding a copyright symbol into an print image which can survive against print-scan process. Copyright protection is a challenging problem specially for printed image document. Digital watermarking is one of the most efficient method to protect an image from unauthorized use. In this paper we propose an inexpensive hardcopy watermarking technique which can survive print-scan transformation as well as small degree of geometric distortion. Experimental result shows the effectiveness of the proposed watermarking scheme. The proposed scheme could be used as an inexpensive method of copyright protection for printed document like passport, id card etc.
\end{abstract}

\section{INTRODUCTION}

Traditional watermarks are visual patterns embedded in a sheet of paper. These watermarks are produced by creating a variation in thickness of paper fiber during the wet-phase of paper making. The embedded design is imperceptible under normal viewing of either side but when the paper is held up to a light source the visual pattern is clearly visible. Security-printing products are commonly found in many important day-to-day applications ranging from identity cards and driving licenses to certificates and passports. Many security documents are subject to fraudulent reproduction, adulteration and theft.Attempted frauds include total book counterfeiting,photograph substitution,page substitution, and expiry date tampering.

Recent years have seen a significant research effort in digital watermarking and data hiding techniques. Unlike traditional watermarks, a majority of these techniques focus on embedding and extracting data from electronic content and not from a hardcopy paper document. A very few number of techniques for embedding visual or non visual data in hardcopy documents have been proposed. Typically,the techniques are specific to the type of document and its content. Examples of this class of techniques include methods that embed information in hardcopy text documents through embedding information in printed halftone images[1][2][3][4] and methods for embedding information in images that are designed to be robust to the printing process[5][6]. Usually these techniques require to capture the printed document in electronic form,from which the embedded data may be recovered or visible pattern may be revealed through suitable processing.

There are two types of attack that may destroy the watermark information, namely deliberate attack and accidental attack. For softcopy image watermarking,the attacks may be introduced deliberately through different signal processing techniques on the other hand image compression, image transmissions in noisy channels are the possible attack, which may happen accidentally. For Hardcopy image watermarking the possible attacks, which may take place unintentionally are changed ink level of the printer, slide rotation of the paper, contrast adjustment of the scanner, print-scan transformation etc. One of the possible attacks that can be involved deliberately on the watermarked by the creation of wrinkled as by crushing or folding. Y.J.Song proposes a robust watermarking scheme for forgery detection in printed materials[7]. C.Y.Lin proposes a model for print and scan without any countermeasure[8]. L.Yu proposes another model for printscan and also proposes a countermeasure[9]. But details description about the watermark bit insertion and extraction process are not available in those published literature.

In this paper We are proposing a simple and easily implementable algorithm for hardcopy image watermarking using DCT . We have suggested a mechanism for choosing DCT coefficient in order to insert watermark. A guide line is also given, how the methodology could be used for a different combinations of printer and scanner. It has been shown that proposed algorithm is working equally well for different pair of printer and scanner. In the present article section 1 describes the overall print-scan process and also different aberration that may happen during print-scan process and how the proposed method can overcome those difficulties. In section 2 ,we propose print-scan transformation technique for a pair of printer and scanner. Section 3 describes a simple hardcopy image watermarking technique. Experimental results are shown in Section 4. Finally, concluding remarks are given in section 5 .

\section{PRINT-SCAN PROCESS}

When printed document which is generated from gray scale digital data is scanned, the resulting scanned data (soft) contains digital value which is quite different from that of digital data from which print document is generated. These differences may not be significant to naked eyes and visually noticeable. The other type of aberration normally invisible to human eye which may occur during scanning includes a small degree of rotation, scaling, cropping due to error in non-exact placing of scanning document. This may pose some 
registration problem when we try to match the corresponding pixels between original documents and the scanned document.

\section{A. Printing Process}

1) Halftoning Process: Most of the image reproduction devices, particularly the printing devices, have restricted capability of printing few colors, while the digital image mostly consists of millions of colors. In the gray scale case, the digital image consists of 256 different shadows of gray, while the black and white printers normally use only one colored ink, i.e. black. These 256 levels of gray should somehow be represented by the black color and the white substrate and this is implemented via halftoning process.

Halftoning is a process that converts a digital gray scale image to a bi-level image while attempting to preserve its visual appearance. Halftoning works by exploiting the low pass characteristics of the human visual system. This is possible because of spatial integration performed by our eyes. Ideally, a local spatial average of the bi-level image generated by the halftoning process at each spatial location closely approximates the original gray value of the image. In halftoning process, a halftone cell is generated by varying the distribution of black dot which corresponds to a particular gray value. There are many different halftoning algorithms adapted to various printing technologies and applications. Three common methods for generating digital halftoning images are namely 1.Patterning 2.Dithering 3.Error diffusion[10]. Patterning is one of the best approaches to generate halftone cell.

2) Patterning: It generates an image from the source image which is of higher spatial resolution than the source image. The number of halftone cells of the output image is the same as the number of pixels of the source image. In patterning technique, each gray value of the digital image is represented by its halftone cell which is nothing but a matrix with variable number of black dots.

$$
\begin{aligned}
& B_{n}= \\
& {\left[\begin{array}{cl}
4 \times B_{-}\{n / 2\}+3 \times U_{-}\{n / 2\} & 4 \times B_{-}\{n / 2\}+1 \times U_{-}\{n / 2\} \\
4 \times B_{-}\{n / 2\} & 4 \times B_{-}\{n / 2\}+2 \times U_{-}\{n / 2\}
\end{array}\right]}
\end{aligned}
$$$$
\text { where } n \geq 2
$$

Equation(1) represents the general equation for generating patterning matrix for halftone cell[11]. Depending upon the value of $n$, different size of patterning matrix which depicts the different gray level range can be generated, eg. when $n=k$ then $(k \times k)+1$ different gray levels can be generated. Based on human visual characteristics $B \_2$ and $B \_3$, which are second order and third order patterning matrix, has been suggested as follows.

$$
B \_2=\left[\begin{array}{ll}
3 & 1 \\
0 & 2
\end{array}\right] \quad B \_3=\left[\begin{array}{ccc}
7 & 2 & 6 \\
4 & 0 & 1 \\
3 & 8 & 5
\end{array}\right]
$$

Using these two primitive matrices the higher order matrix is generated. From $B \_2$ one can generate 5 gray level matrix staring from 0 to 4 . Here $0,1,2,3$ are four distinct positions to filled up by black dots to increase the gray level. When no black dot is placed within the matrix,it could be used to represent an absolute white region which can be defined as first gray level. When position 0 is filled up with a black dot then we call that the second gray level is generated. When positions 0 and 1 both are filled up then third gray level is generated and so on. Accordingly, when all the positions are filled up then fifth gray level is generated. Similarly using $B \_3$, one can generate 10 gray levels. Table I represents the matrix to generate 65 gray levels which is generated using $B_{-} 2$ and $B_{-} 4$ matrix. Any patterning matrix of any order could be generated from the primitive matrix like $B_{2}, B_{3}, B_{5}$ etc.

TABLE I

GENERATION OF MATRIX B_8

B_8 $=$\begin{tabular}{|c|c|c|c|c|c|c|c|}
\hline 63 & 31 & 55 & 23 & 61 & 29 & 53 & 21 \\
\hline 15 & 47 & 07 & 39 & 13 & 45 & 05 & 37 \\
\hline 51 & 19 & 59 & 27 & 49 & 17 & 57 & 25 \\
\hline 03 & 35 & 11 & 43 & 01 & 33 & 09 & 41 \\
\hline 60 & 28 & 52 & 20 & 62 & 30 & 54 & 22 \\
\hline 12 & 44 & 04 & 36 & 14 & 46 & 06 & 38 \\
\hline 48 & 16 & 56 & 24 & 50 & 18 & 58 & 26 \\
\hline 00 & 32 & 08 & 40 & 02 & 34 & 10 & 42 \\
\hline
\end{tabular}

From our above discussion, the final size of an image generated from the halftoning technique will have a size equal to the original image size multiplied by the order of the matrix. The order of the patterning matrix depends on the number of gray level present in the original image. For large number of gray levels like 256, the order of the patterning matrix necessary to fully represent the image is 16 and corresponding halftoned image size is 16 times of the original image. This required a huge computation time and in order to reduce the computation time considerably reduced gray level representation of the original image into its halftoned form. For e.g., one may consider 64 levels in place of 256 gray levels present in the original image. This considerably saves the memory space and computation time without appreciable loss of quality. Under this circumstance, the correspondence between the gray level $(x)$ of the original image and the cell no of its corresponding halftone representation (reduced gray level range) is represented by equation (2).

$$
\begin{aligned}
& \text { Cellno }=((256-x) /(256 /((n \times n))) \\
& \text { where } 0 \leq x \leq 255
\end{aligned}
$$

\section{B. Scanning Procedure:}

Scanner is used to generate a soft version from the hard version of a document. Flatbed scanner is one of the most popular device in this respect. During scanning, document is placed properly on flat glass plate and the scanning light reflects from the document is captured by CCD scanner, which generates the equivalent digital signal proportional to the gray level of the different points in the document.

At the time of scanning, special care should be taken to avoid geometric distortion like scaling, cropping and rotation. If we use the patterning technique to print a gray scale image, then halftoned image size is $n$ times that of the original image size. Scanning resolution is so selected such that its value is equal to the ratio of the printing resolution and order of the patterning matrix,eg., if the printing resolution is $600 \mathrm{dpi}$ and 
order of patterning matrix is 8 then set the scanning resolution $75 \mathrm{dpi}(600 / 8)$. Cropping can be avoided by using magnified version of the soft document. Rotation of the document is another major distortion at the time of scanning. It can be minimized but can not be made zero even with best care. To handle the rotation problem in the watermarking scheme, one should use either a marker to calculate the rotational angle or can use watermarking scheme in RST invariant domain like fourier-mellin transformation[12]. L.Yu suggested a marker model to calculate the rotational angle by morphology based contour tracing[9]. Here we are proposing another efficient scheme to calculate the rotational angle as well as detection technique of the source image.

In this respect, we are using a dense black cross marker in the form of 'L' along with the source, printed as composite image. Marker is placed both parallel and few pixels away to the left and bottom border line of the source image. Fig.1(a) shows the rotated version of the composite image in the clockwise direction. After scanning, the composite image is segmented [13]into two parts, "marker" and "source image". Two extreme points of the marker in vertical direction are represented by $(\mathrm{X} 1, \mathrm{Y} 1)$ and $(\mathrm{X} 2, \mathrm{Y} 2)$. Similarly, two extreme points in the horizontal direction are represented by $(\mathrm{X} 2, \mathrm{Y} 2)$ and $(\mathrm{X} 3, \mathrm{Y} 3)$ which is shown in Fig.1(b). From those coordinate points, the angle of rotation and its sense could be determined using equation(3) and equation(4).

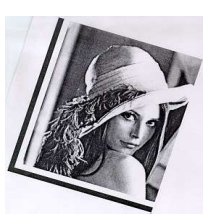

(a)

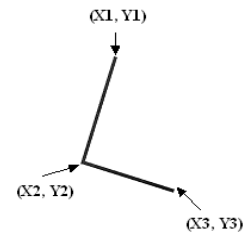

(b)

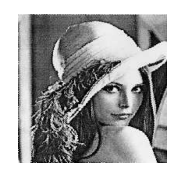

(c)
Fig. 1. (a)Image posted with marker (a)After segmentation of marker portion (b) Extracted main image after rotation

$$
\begin{gathered}
m=\tan (\theta)=(Y 2-Y 1) /(X 2-X 1) \\
\Theta=\tan ^{-1}(m)
\end{gathered}
$$

After rotating the composite image in opposite direction, source image could be determined by cropping space between the marker and source image which is shown in Fig.1(c). Hole effects[14] and artifacts are the possible attack that may degrades the quality of the image after rotation. So interpolation technique is used to remove the hole effects like spline interpolation, cubic interpolation or neighborhood operations etc.

\section{PROPOSED GUIDE LINE FOR MEASURING A PRINT-SCAN TRANSFORMATION}

A tuning mechanism is used to measure print-scan transformation using one which can correlate two images obtained before printing and that of obtained after scanning. The block diagram of overall transformation process is shown in Fig.2.
$T_{1}$ and $T_{2}$ are the transformation which occurred at the time of halftoning to printing and printing to scanning respectively. Total transformation $T$, is equivalent to $T_{1} * T_{2}$, which can be represented by equation(5).

$$
Y_{i}=T\left(X_{i}\right), i=0,1, \cdots 255
$$

$X_{i}=$ Input gray value before printing

$Y_{i}=$ Output gray value after scanning

Let, $P$ represents the inverse transformation function of $T$.

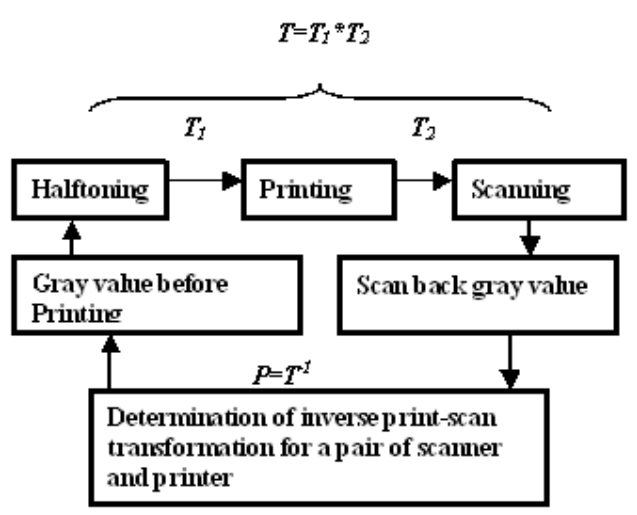

Fig. 2. The block diagram of Print-scan transformation.

To estimate the possible form of transformation function $T$, a synthetic image which contains different strips of known gray level value ( 0 to 255 ) is used. This is converted to the halftone version using the technique mentioned above and then the corresponding printed document is generated. Now, the printed document with different gray level strips is scanned to generate corresponding image output that contains similar strips of different gray level. These gray level values will be different from that of the source document because transformation $T_{1}$ has been occurred during halftoning process and transformation $T_{2}$ has been occurred during scanning process. Now, we can compute the composite transformation $T$, resulting for a particular combination of printer and scanner, and it can be estimated by using the popular polynomial interpolation technique which is as shown as equation(6).

$$
\begin{aligned}
& Y_{i}=\sum_{j=0}^{r} a_{j} X_{i}^{j}, i=0,1, \cdots 255 \\
& \quad a_{j} \text { are Coefficients. }
\end{aligned}
$$

In our experiment, we have used two different combinations of printer-scanner namely Pair 1 (HP LaserJet 1200 series, HP ScanJet 4C) and Pair 2 (HP LaserJet 1200 series, HP ScanJet 8250 ) to test the validity of this kind of technique. It has been found that polynomial up to $4^{t h}$ degree (when $\mathrm{r}=4$ ) is sufficient for the present purpose. Table II represents the values of the coefficient for two different combinations of printerscanner, Pair 1 and Pair 2. The interpolation curve (we call it $T$-curve) for Pair 1 is shown in Fig.3(a) and corresponding inverse transformation curve (we call it $P$-curve) is shown in Fig.3(b). 
TABLE II

COEFFICIENTS VALUES FOR DIFFERENT PAIR

\begin{tabular}{|c|c|c|}
\hline Coefficients & Pair 1 & Pair 2 \\
\hline$a_{4}$ & $-1.8262 \times 10^{-7}$ & $-2.7614 \times 10^{-7}$ \\
\hline$a_{3}$ & $5.1239 \times 10^{-5}$ & $1.4932 \times 10^{-4}$ \\
\hline$a_{2}$ & 0.0039 & -0.0198 \\
\hline$a_{1}$ & -0.4462 & 0.7276 \\
\hline$a_{0}$ & 30.6644 & 45.8628 \\
\hline
\end{tabular}

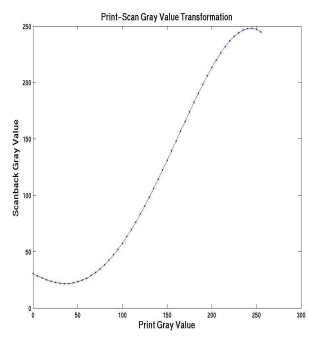

(a)

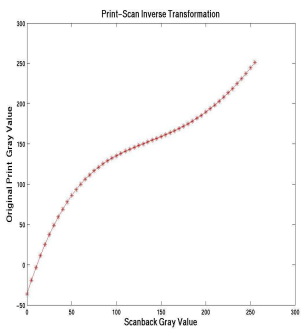

(b)
Fig. 3. (a) $T$-curve.(b) $P$-curve.

\section{WATERMARKING}

Watermarking is a technique by which a marker data or logo could be hidden inside a cover signal/image imperceptibly. In particular, there are two types of watermarking scheme available in the literature, one is visible watermarking and another is invisible watermarking. In this work we have adapted second type. To develop an efficient invisible watermarking scheme, special care should be taken on a) image degradation after watermarking is minimum b) it is sufficiently robust against deliberate and non deliberate attack c) quality of the extracted watermark is close to the inserted one d) low cost implementation. In the case of watermarking for printed document one of the major non deliberate attack is print-scan transformation. In this type of watermark the secret watermark can be inserted in the source image ( before converted to halftone ) or in the halftone image. The literature on watermarking for printed document is not rich. Some of the work in the printed document published recently [1][2][3][4] used halftone version as source image for watermarking. But in the proposed work, watermark information is inserted into the transformed version ( DCT ) of the cover image. There are some other work that uses transform domain for watermarking like DFT [15].

\section{A. Watermark Insertion}

The proposed watermarking is based on the DCT [16]. Let I be a gray level image of size $(M \times N)$, where the secret information/image is inserted. Let a logo binary image $\mathbf{W}$ of size $(a \times b)$ is used. If we assume that $M=N$ and $a=b$, then the total image space that could be utilized properly if $M / a$ is an integer number and generally it is $\geq 8$ for $(8 \times 8)$ Discrete Cosine Transformation. If $M / a$ and $N / b$ is not a pure integer then some row or column padding is done to match properly the dimension of logo with that of cover image.

The original image $\mathbf{I}$ is partitioned into number of $(8 \times 8)$ blocks and DCT is computed for each block. In each block of transformed image, one pixel information ( corresponding to 0 or 1) of logo binary image is inserted. The technique which is used to insert a watermark signal is given in Algorithm1. To insert this information, a proper DCT coefficient is selected in such a way that it can survive the print-scan transformation and its inverse transformation. It is found experimentally that modification of DCT coefficient value, cause visual distortion of the original image of different degree, based on the component selected for modification. The modification of lower frequency component causes greater distortion and also non diagonal frequency component causes asymmetric distortion, which is visually disturbing. Considering those facts, we have selected diagonal frequency component $f(k, k)$, where $k \geq 4$ for minimal distortion. At the time of watermark insertion, one can use $\alpha$ times of the global maximum $\left(W_{\max }\right)$ of the frequency component $f(k, k)$, so that in the watermarked image dynamic range of this frequency component will be larger than its original dynamic range. It will help to detect the watermark bit from a highly distorted scan-back image. The value of $\alpha$ will be chosen depending on the allowed visual degradation of the watermarked image. Watermark insertion and extraction in different frequency component is given in the section $\mathrm{v}$.

\section{B. Watermark Extraction}

The reverse procedure is followed to extract watermark information. The logo image $\mathbf{W}^{\prime}(a \times b)$ is generated from the scan-back image $\mathbf{S}(M \times N)$ using the Algorithm2. This algorithm is applied to the scan-back image after identification of the image which is discussed in section II and gray value transformation by using p-curve which is discussed in section III.

\section{Similarity Measurement}

In this proposed technique measurement of the quality of the extracted watermark symbol is estimated using popular image correlation technique like Normalized Cross Correlation (NCC) which can be expressed as follows.

$$
N C C=\sum_{a} \sum_{b} \mathbf{W}(a \times b) \mathbf{W}^{\prime}(a \times b) / \sum_{a} \sum_{b}[\mathbf{W}(a \times b)]^{2}
$$

Here extracted watermark $\mathbf{W}^{\prime}$ is compared with the original watermark $\mathbf{W}$. The maximum value of NCC will be 1 when both are same, otherwise closer the value of NCC is the better result.

\section{EXPERIMENTED RESULTS}

We describe our algorithm for hiding $(22 \times 20)$ binary image $(\log 0)$ into two gray level cover images lena $(222 \times 208)$ and boat $(256 \times 256)$, $8 \mathrm{bit} / \mathrm{pixel}$. In our experiment, we have used patterning matrix of order 8 which is computationally simple and efficient. From our above mentioned watermarking scheme some experimented results are produced where most of the bits are successfully extracted. The experiment has been carried on two different frequency components of DCT block, $\mathrm{f}(4,4)$ and $\mathrm{f}(5,5)$. The result is given in Table III. Watermark insertion as well as extraction in different frequency component for different images and image transformation in different level are shown in Fig.4, Fig.5, Fig.6, Fig.7. 


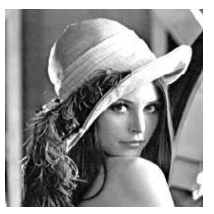

(a)

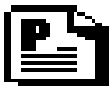

(b)

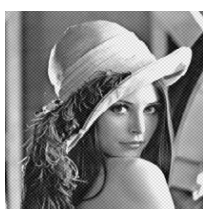

(c)

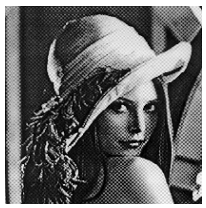

(d)

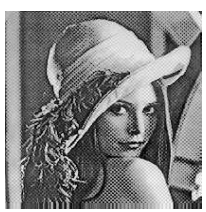

(e)

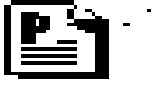

(f)

Fig. 4. (1,TABLE III ) (a). Original image. (b). Logo image. (c).watermarked image with $\mathrm{f}(4,4)$ frequency component as location of insertion (d).scan-back image (e).scan-back image after inverse print-scan transformation F.Extracted hidden image.

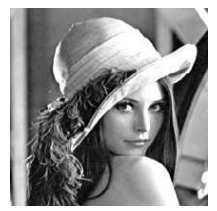

(a)

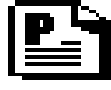

(b)

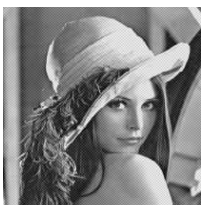

(c)

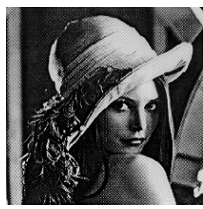

(d)

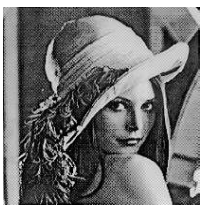

(e)

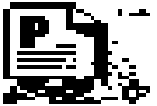

(f)

Fig. 5. (2,TABLE III )(a). Original image. (b). Logo image. (c).watermarked image with $f(5,5)$ frequency component as location of insertion (d).scan-back image (e).scan-back image after inverse print-scan transformation F.Extracted hidden image.

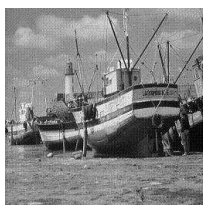

(a)

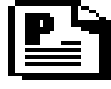

(b)

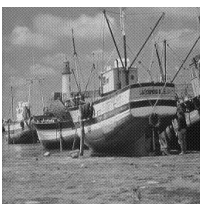

(c)

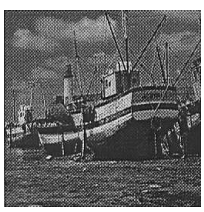

(d)

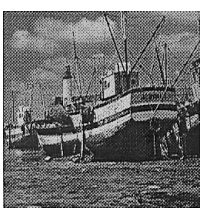

(e)

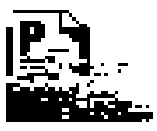

(f)

Fig. 6. (3,TABLE III ) (a). Original image. (b). Logo image. (c).watermarked image with $f(4,4)$ frequency component as location of insertion (d).scan-back image (e).scan-back image after inverse print-scan transformation F.Extracted hidden image.

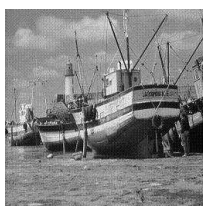

(a)

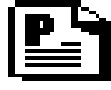

(b)

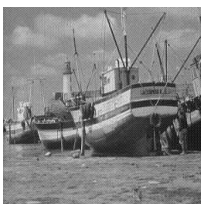

(c)

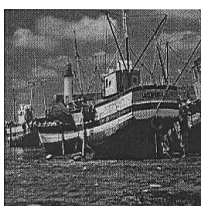

(d)

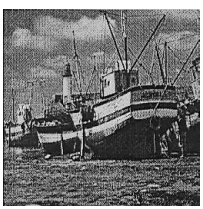

(e)

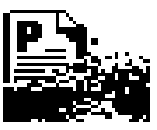

(f)

Fig. 7. (7,TABLE III )A. Original image. B. Logo image. C.watermarked image with f(5,5) frequency component as location of insertion D.scan-back image E.scan-back image after inverse print-scan transformation F.Extracted hidden image.

TABLE III

WATERMARK RESULT ON THE STANDARD IMAGE LENA AND BOAT.

\begin{tabular}{|c|c|c|c|c|c|}
\hline $\begin{array}{c}\text { Exp } \\
\text { No }\end{array}$ & Image & $\begin{array}{c}\text { Frequency } \\
\text { component }\end{array}$ & rotation & NCC & Resource \\
\hline 1 & lena & $\mathrm{f}(4,4)$ & 0.238852 & 0.994485 & Pair1 \\
\hline 2 & lena & $\mathrm{f}(5,5)$ & 0.0 & 0.784926 & Pair1 \\
\hline 3 & boat & $\mathrm{f}(4,4)$ & 0.209980 & 0.6598 & Pair 2 \\
\hline 4 & boat & $\mathrm{f}(4,4)$ & 0.0000 & 0.8021 & Pair 2 \\
\hline 5 & lena & $\mathrm{f}(4,4)$ & 0.238852 & 0.928309 & Pair 2 \\
\hline 6 & boat & $\mathrm{f}(5,5)$ & -0.2062803 & 0.798953 & Pair 2 \\
\hline 7 & boat & $\mathrm{f}(5,5)$ & 1.259684 & 0.6000 & Pair 2 \\
\hline 8 & lena & $\mathrm{f}(5,5)$ & .479695 & 0.5200 & Pair 2 \\
\hline
\end{tabular}

\section{CONCLUSION}

A model of print-scan and watermarking are proposed in this paper. Proposed technique describes robust and semi blind digital image watermarking in frequency domain which is computationally efficient. From the experimented result we can conclude that the proposed algorithm is also effective for small degree of document rotation. Proposed technique has several advantages. The halftoning technique and watermarking procedure both are easy to implement. Secondly,based on the requirement for degree of degradation and robustness in a particular watermarking process, we can select the different frequency component accordingly. The proposed scheme can hide logo binary image as well as gray scale image into a gray scale image. Further research work is being carried out for inserting barcode logo image into a halftone gray scale and color documents.

\section{REFERENCES}

[1] Ming Sun Fu and Oscar C.Au, "Data hiding by smart pair toggling for halftone images," Proc. of IEEE Int. Conf On Acoustics,Speech and Signal Processing, vol. 4, pp. 2318-2321, June 2000.

[2] Ming Sun Fu and Oscar C. Au, "Data hiding watermarking for halftone images," IEEE Transactions on Image Processing, vol. 11, no. 4, pp. 477-484, April 2002.

[3] Hae Yong Kim and Amir Afif, "A secure authentication watermarking for halftone and binary images," International Journal Imaging Systems and Technology, vol. 14, no. 4, pp. 147-152, 2004.

[4] Ming Sun Fu and Oscar C.Au, "Halftone image data hiding with intensity selection and connection selection," Signal Processing:Image Communication, vol. 16, pp. 909-930, 2001.

[5] Adnan M.Alattar, "Smart images using digimarc's watermarking technology," 12th Unternational Symposium on Electronic Imaging, vol. 3971, no. 25, January 2000. 


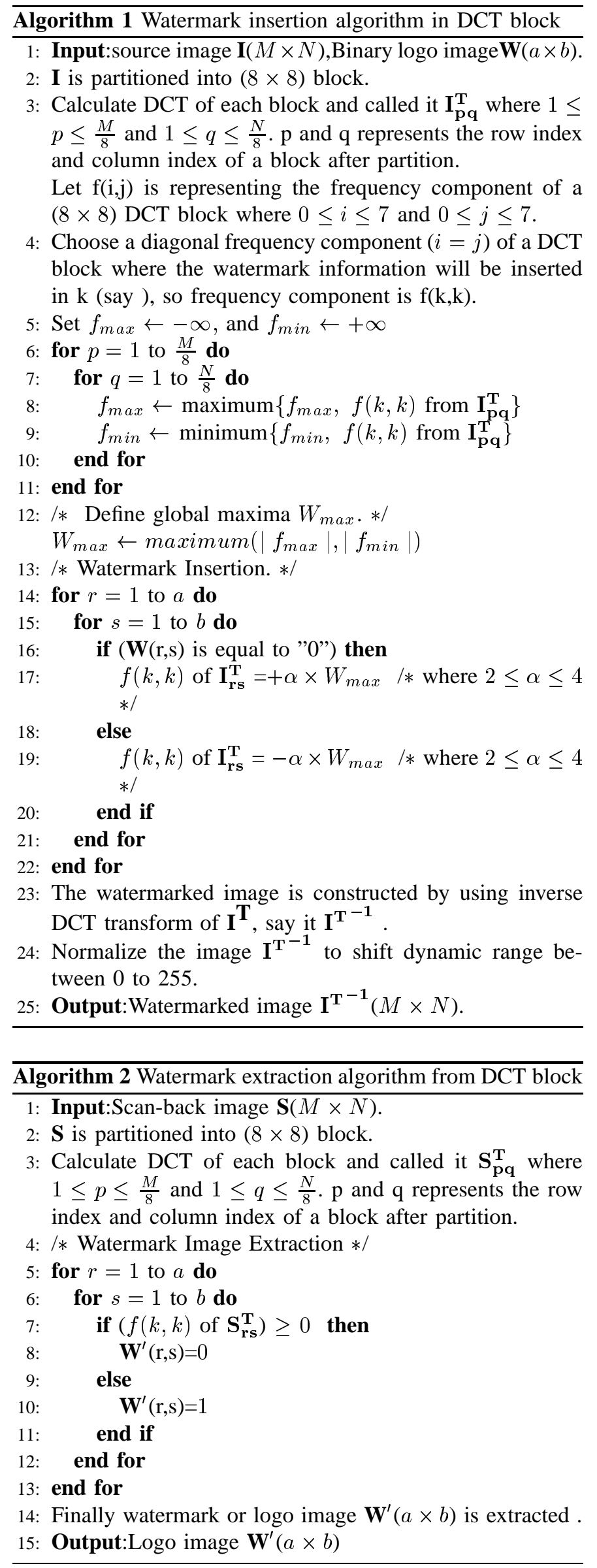

[6] S.Decker, "Engineering considerations in commercial watermarking," IEEE Comm.Mag., vol. 39, pp. 128-133, August 2001.

[7] Y.J.Song, R.Z.Liu, and T.N.Tan, "Digital watermarking for forgery detection in printed materials," Advanced in Multimedia Information Processing-PCM 2001:Second IEEE Pacific rim Conference on Multimedia.

[8] Ching-Yung Lin and shih Fu Chang, "Distortion modeling and invariant extraction for digital image print-and-scan process," International Symposium on Multimedia Information Processing (ISMIP), Dec 1999.

[9] Longjiang Yu, Xiamu Niu, and Shenghe Sun, "Print-and-scan model and the watermarking countermeasure," Image and vision Computing, , no. 23 , pp. $807-814,2005$.

[10] "www.geocities.com/researchtriangle/thinktank/5996/techpaps/introip/ manual04.html,"

[11] "www.cs.uvm.edu/ xqzhu/cs295/lectures/dhalftoning.ppt," .

[12] Ching-Yung Lin, Min Wu, Jeffrey A.Bloom, Ingemar J.Cox, Matt L.Miller, and Yui Man Lui, "Rotation,scale, and translation resilient watermarking for images," IEEE TRANSACTIONS ON IMAGE PROCESSING, vol. 10, no. 5, pp. 767-782, May 2001.

[13] Digital Image Processing, R.C.Ganzalez and R.E.Woods, Pearson education edition, 2003.

[14] Hoo-Cheng, Liu Shou-Yiing Hsu, Mu-Hwa Chen, Yih-Farn Wang, TsuFeng Kuo, and Chaoyin Chien, "On the hole effect of image rotation algorithms," Proc.Natl.Sci.Counc.ROC(A), vol. 24, no. 3, pp. 186-194, 2000.

[15] Avi Levy and Doron Shaked, "A transform domain hardcopy watermarking scheme," HP Laboratories Israel, November 2001.

[16] E.Koch and J.Zhao, "Towards robust and hidden image copyright labeling," In:Proc of 1995 IEEE Workshop on Nonlinear Signal and Image Processing,Neos Marmaras, greece., 20-22 June 1995. 